\title{
Relaçáo da Sobrecarga do Cuidador, Perfil Funcional e Qualidade de Vida em Crianças com Paralisia Cerebral
}

\author{
Relationship Among Caregivers Burden, Functionality and Quality of Life in Children with Cerebral Palsy
}

\author{
Ana Paula Machi Barbosa, Tamires Nicoly Zampa ${ }^{2}$, Cristina Iwabe ${ }^{3}$, \\ Maria Angélica da Rocha Diz
}

\begin{abstract}
RESUMO
Objetivo. Avaliar a influência do comprometimento motor na qualidade de vida de crianças com Paralisia Cerebral (PC) e a sobrecarga do seu cuidador. Método. Foram avaliadas 12 crianças, seis do gênero feminino e seis do masculino, com diagnóstico clínico de PC quadriplégicos e diplégicos, na faixa de 5 a 12 anos de idade, através das escalas Avaliação de Qualidade de Vida (AUQEI), versão brasileira da Burden Interview (BI), e Gross Motor Function Classification System (GMFCS). Utilizou-se o coeficiente de correlação de Pearson para analisar a correlação entre as variáveis. Resultados. A classificação do GMFCS variou dos níveis II ao $\mathrm{V}$, sendo mais freqüente o nível IV (50\%); o escore da escala AUQEI apresentou média de 46,75 (máximo de 78); na BI verificou-se escore médio de 24,16 (máximo de 88). Observou-se correlação forte significativa somente entre o tipo de PC e o GMFCS; houve correlação significativa intermédia entre tipo de GMFCS e AUQEI; não houve correlações entre as demais variáveis. Conclusáo. $\mathrm{O}$ comprometimento motor da criança com $\mathrm{PC}$ não apresenta relaçáo preditiva entre o estresse e a sobrecarga do cuidador; nem táo pouco com a qualidade de vida relatada por essas crianças.
\end{abstract}

Unitermos. Paralisia Cerebral, Qualidade de Vida, Cuidador.

Citaçáo. Barbosa APM, Zampa TN, Iwabe C, Diz MAR. Relação da Sobrecarga do Cuidador, Perfil Funcional e Qualidade de Vida em Crianças com Paralisia Cerebral.

\begin{abstract}
Objective. To evaluate the influence of motor impairment in quality of life in children with Cerebral Palsy (CP) and the burden of their caregivers. Method. We evaluated 12 children, six females and six males with a clinical diagnosis of quadriplegic and diplegic $\mathrm{CP}$, ages 5 to 12 years old, with scales Assessment of Quality of Life (AUQEI), brazilian version of the Burden Interview (BI), and Gross Motor Function Classification System (GMFCS). For statistical analysis we used the Pearson correlation coefficient to determine the correlation between variables. Results. The classification of GMFCS levels ranged from II to V, more frequent level IV (50\%), the scale AUQEI score had an average of 46.75 points (maximum 78 ); and the BI showed mean score 24.16 (maximum 88). There was a strong significant correlation only between the type of CP and GMFCS; intermediate significant correlation between GMFCS and AUQEI; there was no correlation between the other variables. Conclusion. The motor impairment of children with CP is not related predictor of stress and caregiver burden, nor with the quality of life reported by these children.
\end{abstract}

Keywords. Cerebral Palsy, Quality of Life, Caregiver Burden.

Citation. Barbosa APM, Zampa TN, Iwabe C, Diz MAR. Relationship Among Caregivers Burden, Functionality and Quality of Life in Children with Cerebral Palsy.

\footnotetext{
Trabalho realizado na clínica de fisioterapia do Centro Universitário Padre Anchieta e Faculdade Anhanguera Unidade SBO.

1,2.Fisioterapeuta, Centro Universitário Padre Anchieta, Jundiaí-SP, Brasil.

3.Fisioterapeuta, Doutora em Ciências Médicas Unicamp, Docente do Centro Universitário Padre Anchieta e Veris IBTA Metrocamp - Curso de Fisioterapia, Campinas-SP, Brasil.

4.Fisioterapeuta, Docente do Curso de Fisioterapia - Faculdade Anhanguera

Unidade Santa Bárbara d'Oeste, Santa Bárbara d'oeste-SP, Brasil.
}

Endereço para correspondência: Cristina Iwabe Rua Mata dos Pinhais 61, casa 76, Barão Geraldo CEP 13082-761, Campinas-SP, Brasil. E-mail: crisiwabe@hotmail.com; ciwabe@fcm.unicamp.br

Original Recebido em: 25/07/11 Aceito em: 09/02/12 Conflito de interesses: não 


\section{INTRODUÇÃO}

Paralisia Cerebral (PC) designa um conjunto de sinais decorrentes de uma lesão no sistema nervoso central imaturo, de caráter crônico não progressivo. Tais distúrbios caracterizam-se pela desordem de movimento, tônus e postura ${ }^{1,2}$, ocasionando distúrbios motores diferenciados e classificados de acordo com a parte comprometida do corpo: hemiplegia, diplegia ou quadriplegia ${ }^{3,4}$.

Existem várias causas etiológicas relacionadas à PC, como desenvolvimento congênito anormal do cérebro, anóxia cerebral perinatal ou infecções cerebrais ${ }^{5,6}$. O diagnóstico deve ser baseado em uma história clínica completa, na avaliação física e neurológica da criança ${ }^{6}$.

Diante dos diferentes quadros clínicos observados, diversas modalidades de tratamentos objetivam promover saúde, independência funcional e, sobretudo, qualidade de vida a crianças com $\mathrm{PC}^{7,8}$.

Segundo a Organização Mundial de Saúde, a qualidade de vida é definida como a percepção do indivíduo de sua posição na vida, contexto e valores culturais nos quais vive, e a relação com suas metas, objetivos e expectativas. É um amplo conceito que incorpora a saúde física da pessoa, status psicológico, nível de independência, relacionamento, inserçâo social e sua relação com as características do ambiente?.

As alteraçóes encontradas em crianças com PC como, variação do tônus muscular, persistência dos reflexos primitivos, e a espasticidade ocasionam padróes atípicos de postura e movimentos que comprometem o seu desempenho funcional motor e atividades de rotina diária, como tomar banho, alimentar-se, vestir-se e locomover-se ${ }^{8}$.

Essas alteraçóes levam o cuidador a alterar sua rotina a fim de propiciar adequadas condiçóes de vida à criança. A saúde psicológica, física e mental dos cuidadores pode ser fortemente influenciada pelo comportamento da criança e pela demanda do seu cuidador. Assim, a tarefa de assistir crianças com diagnóstico de PC, aliado ao aumento de responsabilidades que essa função promove, pode levar ao cansaço, isolamento, sobrecarga e estresse dos cuidadores ${ }^{10}$.

Em virtude desses aspectos, este estudo objetivou analisar a influência do comprometimento motor na qualidade de vida de crianças com diagnóstico de PC e a sobrecarga do seu cuidador.

\section{MÉTODO}

\section{Amostra}

A pesquisa foi realizada na clínica de fisioterapia do Centro Universitário Padre Anchieta (Unianchieta) em Jundiaí e na Faculdade Comunitária de Limeira.

Foram incluídas crianças com diagnóstico clínico de PC, tipos clínicos quadriplégicos e diplégicos, na faixa etária de 5 a 12 anos de idade, ambos os gêneros, com cognição preservada para compreensão de ordens simples, e seus respectivos cuidadores.

$\mathrm{Na}$ coleta de dados foram utilizados três protocolos: A Avaliação de Qualidade de Vida (AUQEI) - uma escala validada, de total confiabilidade e de fácil aplicação em crianças de 4 a 12 anos; a versão brasileira da Burden Interview, aplicada nos cuidadores, com 22 questóes relacionadas à sobrecarga do cuidador; e Gross Motor Function Classification System (GMFCS) que avalia o nível de funcionalidade de crianças com PC.

Este estudo foi previamente aprovado pelo Comitê de Ética em Pesquisa do Centro Universitário Padre Anchieta, sob parecer número 058/2010.

\section{Procedimento}

Após aprovação do estudo, os cuidadores das crianças selecionadas foram informados acerca dos objetivos da pesquisa, autorizando a sua participação e das crianças, através da assinatura do Termo de Consentimento Livre e Esclarecido, conforme resolução 196/96 do Conselho Nacional de Saúde do Ministério da Saúde.

A seguir foi aplicado o questionário AUQEI e a escala GMFCS nas crianças com diagnóstico de PC, e a Burden Interview nos respectivos cuidadores.

\section{Análise Estatística}

$\mathrm{Na}$ análise estatística dos dados foi utilizado o coeficiente de correlação de Pearson (r) para verificação da associação entre as variáveis. Foram realizadas correlaçóes entre: tipo clínico de PC e nível do GMFCS; tipo clínico de PC e AUQEI; tipo clínico de PC e Burden Interview; AUQEI e Burden Interview; nível de GMFCS e AUQEI; e nível de GMFCS e Burden Interview.

$\mathrm{O}$ valor de $\mathrm{r}$ pode variar de $-1 \mathrm{a}+1$, sendo que quanto mais próximo desses valores, mais forte a associação entre as variáveis, ou seja, escore zero indica ausência 
de correlação; $r>0,8$ - correlação forte; $0,8<\mathrm{r}<0,6$ correlação intermediária; $r \leq 0,6$ - correlação fraca. Nível de significância $\mathrm{p}<0,01$.

\section{RESULTADOS}

Foram avaliadas 12 crianças, com idades entre 5 a 12 anos (média de 8,58 anos), seis do gênero feminino e seis masculinos, seis quadriplégicos e seis diplégicos.

Em relação ao GMFCS, este variou do II ao V, sendo mais freqüente o nível IV (50\%). O nível da qualidade de vida das crianças, mensurada pela escala AUQEI, apresentou média de pontos de 46,75, sendo que o escore máximo possível é de 78 (expressão de muito feliz). Para a sobrecarga dos cuidadores, verificou-se que o escore médio obtido foi de 24,16, sendo que o escore máximo possível é de 88 (máxima sobrecarga) (Tabela 1).

Analisando a correlação entre as variáveis observaram-se valores fortes e significativos entre o tipo clínico de PC e o GMFCS. Houve também correlação intermediária entre GMFCS e AUQEI. Não foram observadas demais significâncias (Tabela 2).

\section{DISCUSSÃO}

Dentre os diferentes tipos clínicos de PC, a quadriplegia apresenta pior comprometimento motor ${ }^{8,11-16,17,18}$, com maiores dificuldades para executar as atividades fun- cionais, enquanto o tipo diplégico apresenta níveis de gravidade e dificuldades motoras inversas ${ }^{19}$. Esses relatos são semelhantes aos nossos resultados, apresentando relação entre os maiores níveis de GMFCS e o maior comprometimento do quadro motor (nos quadriplégicos). Os níveis mais baixos, II e III do GMFCS, relacionaram-se aos tipos clínicos com menor gravidade (nos diplégicos).

A qualidade de vida de crianças com condições crônicas severas pode ser influenciada por vários fatores, tais como status socioeconômico, suporte social, características dos pais e das crianças, e capacidade de enfrentamento de problemas ${ }^{5,20}$.

O comprometimento motor da criança com PC pode vir a influenciar na sua qualidade de vida ${ }^{3}$. Tal correlação não foi observada no nosso estudo, visto que o tipo clínico não interferiu na qualidade de vida das crianças. Alguns outros aspectos como nível socioeconômico, funções cognitivas e freqüência de crises epilépticas podem estar envolvidos nesse aspecto, porém não foram analisadas no nosso estudo. Segundo estudos prévios, a associação desses múltiplos fatores pode intervir na qualidade de vida da criança com $\mathrm{PC}^{3,5,20}$.

Em relação à sobrecarga do cuidador, apesar das mães de crianças com PC apresentarem um sofrimento psicológico, logo após a confirmação clínica do diagnóstico, elas parecem ter o bem estar preservado, em conse-

Tabela 1

Perfil das crianças avaliadas, segundo o nivel GMFCS, AUQEI e BI

\begin{tabular}{ccccccc}
\hline CRIANÇAS & TIPO PC & IDADE & GÊNERO & GMFCS & AUQEI & BI \\
\hline 1 & Diplégico & 6 & Feminino & III & 35 & 35 \\
2 & Quadriplégico & 12 & Masculino & IV & 56 & 17 \\
3 & Quadriplégico & 12 & Feminino & IV & 51 & 29 \\
4 & Diplégico & 5 & Masculino & II & 50 & 6 \\
5 & Diplégico & 7 & Masculino & III & 45 & 37 \\
6 & Quadriplégico & 10 & Feminino & IV & 56 & 31 \\
7 & Quadriplégico & 9 & Feminino & IV & 52 & 22 \\
8 & Diplégico & 8 & Feminino & II & 33 & 10 \\
9 & Diplégico & 8 & Feminino & II & 44 & 24 \\
10 & Quadriplégico & 11 & Masculino & IV & 38 & 39 \\
11 & Quadriplégico & 9 & Feminino & IV & 65 & 15 \\
12 & Diplégico & 6 & Masculino & III & 36 & 25 \\
Média & ----- & 8,58 & $-\ldots---$ & ---- & 46,75 & 24,16 \\
\hline
\end{tabular}

GMFCS: gross motor function measure; AUQEI: avaliação de qualidade de vida; BI: Burden Interview 
Tabela 2

Correlaçâo entre as variáveis: tipo clínico de PC, GMFCS, AUQUEI e BI

\begin{tabular}{|c|c|c|c|}
\hline Correlação & Valor de $\mathbf{r}$ & p-valor & Tipo de Correlaçáo \\
\hline Tipo de PC x GMFCS & 0,88 & $0,0001^{*}$ & Correlação forte e significativa \\
\hline Tipo de PC x AUQEI & 0,65 & 0,02 & $\begin{array}{l}\text { Correlaçáo intermediária sem } \\
\text { significância }\end{array}$ \\
\hline Tipo de PC x Burden & 0,13 & 0,68 & $\begin{array}{c}\text { Correlação fraca sem } \\
\text { significância }\end{array}$ \\
\hline AUQEI x Burden & 0,26 & 0,39 & $\begin{array}{l}\text { Correlação fraca sem } \\
\text { significância }\end{array}$ \\
\hline GMFCS x AUQEI & 0,65 & $0,01^{*}$ & $\begin{array}{c}\text { Correlação intermediária - frac } \\
\text { com significância }\end{array}$ \\
\hline GMFCS $x$ Burden & 0,30 & 0,34 & $\begin{array}{c}\text { Correlação fraca sem } \\
\text { significância }\end{array}$ \\
\hline
\end{tabular}

PC: paralisia cerebral; GMFCS: gross motor function measure; AUQEI: avaliação de qualidade de vida; BI: Burden Interview; r: correlaçăo de Pearson

quência de um processo de adaptação psicossocial eficiente, com uma percepção menos negativa da situação por não ter a expectativa de perda tão evidente ${ }^{14,15}$.

Pesquisas relatam que a qualidade de vida das mães de crianças com PC pode ser considerada em média satisfatória. Entretanto, se levarmos em conta os parâmetros para a população geral, a qualidade de vida das mães de crianças com PC é baixa. A mesma ausência de sobrecarga dos cuidadores foi observada em outro estudo, sendo que em alguns casos, fatores como problemas comportamentais da criança, particularmente as interaçôes sociais ${ }^{15,20,21}$, a sua dificuldade de inclusão escolar, ou as condiçôes econômicas desfavoráveis ${ }^{19}$ podem ser preditivos do estresse materno.

Tais estudos corroboram nossos resultados, visto que não houve correlação entre o tipo de $\mathrm{PC}$ e a sobrecarga dos cuidadores. Não foram realizadas análises sócioeconômicas com as famílias para verificar as possíveis variáveis influenciadoras nesse processo, sendo viável a sua pesquisa futura, como parte de orientação familiar a esses cuidadores.

\section{CONCLUSÃO}

O comprometimento motor da criança com PC não apresenta relação preditora com o estresse e a sobrecarga do cuidador, nem tâo pouco com a qualidade de vida relatada por essas crianças. Tendo em vista que a grande parte dos cuidadores são os pais, ao serem questio- nados sobre os cuidados dedicados à criança, verificou-se uma subjetividade das respostas por não relacionarem a demanda dedicada com sobrecarga e sim com o simples fato do cuidar.

O possível estresse do cuidador pode estar relacionado à visão da perspectiva de futuro de suas crianças, podendo interferir na sua qualidade de vida.

\section{REFERÊNCIAS}

1.Chagas PSC, Defilipo EC, Lemos RA, Mancini MC, Frônio JS, Caravalho RM. Classification of Motor Function and Functional Performance in Children with Cerebral Palsy. Rev Bras Fisiot 2008;12;409-16.

http://dx.doi.org/10.1590/S1413-35552008000500011

2.Rocha AP, Afonso DRV, Moraes LS. Relação Entre Desempenho Funcional de Crianças com Paralisia Cerebral e Qualidade de Vida Relacionada à Saúde de Seus Cuidadores. Fisioter Pesq 2008;15:292-7.

3.Christofoletti G, Hygashi F, Godoy ALR. Paralisia Cerebral: Uma análise do Comprometimento Motor Sobre a Qualidade de Vida. Fisioter Mov 2007;20:34-7.

4.Mancini MC, Alves ACM, Schaper C, Figueiredo EM, Sampaio RF, Coelho ZAC, et al. Gravidade da paralisia cerebral e desempenho funcional. Rev Bras Fisiot 2004;8(3):253-60.

5.Camargos ACR, Lacerda TTB, Viana AS, Pinto LRA, Fonseca MLS. Avaliaçấo da sobrecarga do cuidador de crianças com paralisia cerebral através da escala Burden Interview. Rev Bras Saúde Matern Infant 2009;1(9):31-7.

http://dx.doi.org/10.1590/S1519-38292009000100004

6.Assunção Junior FR, Kuczynski E, Sprovieri MH, Aranha EMG. Escala de avaliaçấo de qualidade de vida (AUQEI). Validade e confiabilidade de uma escala para qualidade de vida em crianças de 4 a 12 anos. Arq Neuropsiquiatr 2000;58(1):119-27. 
7.Scazufca M. Brazilian version of the Burden Interview scale for the assessment of burden of care in carrers of people with mental illnesses. Rev Bras Psiquiatr 2002;24(1):12-7.

http://dx.doi.org/10.1590/S1516-44462002000100006

8.Palisano R, Rosenbaum P, Walter S, Russell D, Wood E, Galuppi B. Development and reliability of a system to classify gross motor function in children with cerebral palsy. Dev Med Child Neurol 1997;39(4):214-23.

http://dx.doi.org/10.1111/j.1469-8749.1997.tb07414.x

9.Cury VCR, Mancini MC, Melo AP, Fonseca ST, Sampaio RF, Tirado MGA.

Efeitos do uso de órtese ma mobilidade funcional de crianças com paralisia cerebral. Rev Bras Fisioter 2006;10(1):67-74.

http://dx.doi.org/10.1590/S1413-35552006000100009

10. Ostensjo S, Carlberg EB, Vollestad NK. Motor impairments in young children with cerebral palsy: relationship to gross motor function and everyday activities. Dev Med Child Neurol 2004;46(9):580-9.

http://dx.doi.org/10.1111/j.1469-8749.2004.tb01021.x

http://dx.doi.org/10.1017/S0012162204000994

11.Wood E, Rosenbaum PL. O Gross Motor Function Classification System para a paralisia cerebral: Um estudo de confiabilidade no tempo. Dev Med Child Neurol 2000;42:292-6.

http://dx.doi.org/10.1017/S0012162200000529

http://dx.doi.org/10.1111/j.1469-8749.2000.tb00093.x

12.Rosenbaum PL, Walter SD, Hanna SE, Palisano RJ, Russell DJ, Raina P, et al. O prognóstico para a funçấo motora na paralisia cerebral. Criação de curvas de desenvolvimento motor. Jornal da Associaçăo Médica Americana 2002;288:1357-63.

13.Morris C, Bartlett D. Gross Motor Function Classification System: Impacto e Atualidade. Dev Med Child Neurol. 2004;46:60-5.

http://dx.doi.org/10.1111/j.1469-8749.2004.tb00436.x

http://dx.doi.org/10.1017/S0012162204000751
http://dx.doi.org/10.1017/S0012162204000118

14.Freitas PM, Moreira PPCMS, Rezende LL, Haase VG. Qualidade de vida de mães de crianças com paralisia cerebral. Arquivos Brasileiros de Paralisia Cerebral 2006;2(5):35-9.

15.Freitas PM, Carvalho RCL, Leite MRSDT, Haase VG. Relação entre o stress materno e a inclusão escolar de crianças com Paralisia Cerebral. Arquivo Brasileiro Psicologia 2005;57(1-2):46-57.

16.Morris C, Kurinczuk JJ, Fitzpatrick R, Rosenbaum PL. Do the abilities of children with cerebral palsy explain their activities and participation? Dev Med Child Neurol 2006,48:954-61.

http://dx.doi.org/10.1111/j.1469-8749.2006.tb01265.x

http://dx.doi.org/10.1017/S0012162206002106

17.Morris C, Kurinczuk JJ, Fitzpatrick R, Rosenbaum PL. Reliability of the Manual Ability Classification System for children with cerebral palsy. Dev Med Child Neurol 2006;48:950-3.

http://dx.doi.org/10.1017/S001216220600209X

18.Palisano R, Rosenbaum P, Walter S, Russell D, Wood E, Galuppi B. Gross Motor Function Classification System for Cerebral Palsy. Dev Med Child Neurol 1997;39:214-223.

http://dx.doi.org/10.1111/j.1469-8749.1997.tb07414.x

19.Imm SC, Carlin J, Eliasson AC. Stability of caregiver-reported manual ability and gross motor function classifications of cerebral palsy. Dev Med Child Neurol 2009;52(2):153-9.

20.Morris C, Bartlett D. Gross Motor Function Classification System: impact and utility. Dev Med Child Neurol 2004,46:60-5.

http://dx.doi.org/10.1111/j.1469-8749.2004.tb00436.x

http://dx.doi.org/10.1017/S0012162204000118

21.Mugno D, Ruta L, D’Arrigo VG, Mazzone L. Impairment of quality of life in parents of children and adolecents with pervasive developmental disorder. Health Qual Life Outcomes 2007;27:5-22. 